\title{
Atmospheric DBD Plasma Source in Play for SERS Active Substrates
}

\author{
Taj Muhammad Khan ${ }^{1,2 *}$ and Babar Hussain ${ }^{3}$ \\ ${ }^{1}$ School of Physics, Trinity College Dublin, Ireland \\ ${ }^{2}$ Materials division, Pakistan \\ ${ }^{3}$ Intel Corporation, USA \\ *Corresponding author: Taj Muhammad Khan, School of Physics, Ireland
}

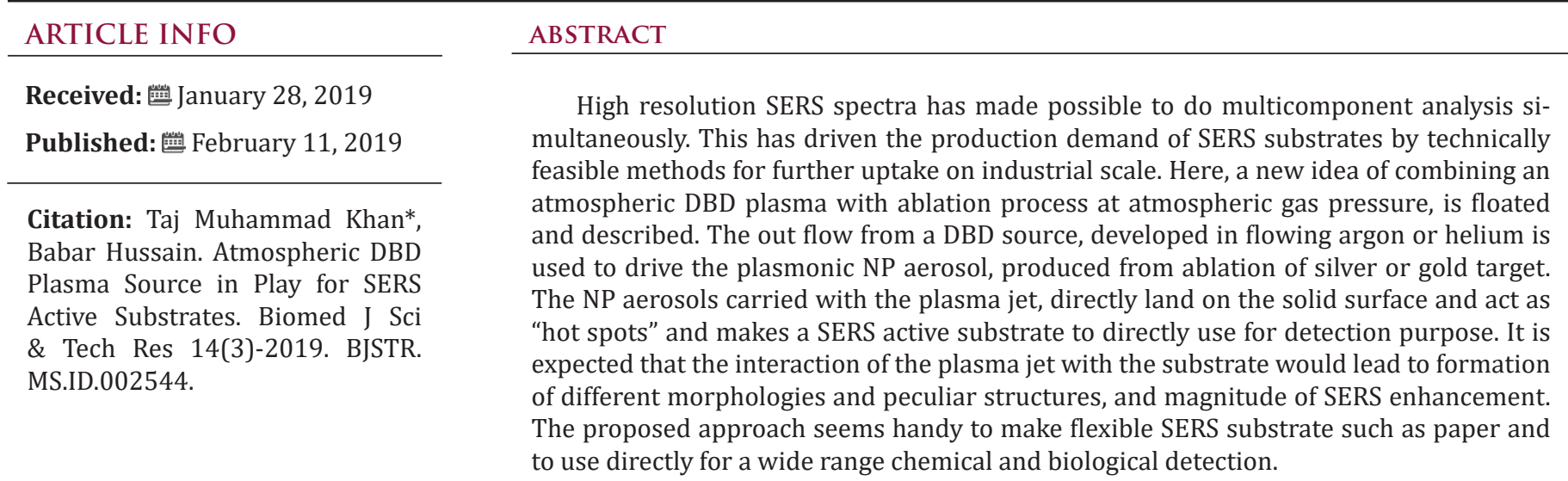

Abbreviations: SERS: Surface-Enhanced Raman Scattering; TCD: Trinity College Dublin; SPR: Surface Plasmon Resonance; EM: Electromagnetic Mechanism; NPs: Nano Particles; APLD: Atmospheric Pulsed Laser Deposition

\section{Introduction}

This mini report describes the idea of an experimental approach, recently floated and executed in the plasma application lab at Trinity College Dublin (TCD). The main aim here is to share this idea with the science community for further investigation, development and practical application. Further objective is to familiarize researchers with the idea and to let them know how this novel approach could be effectively applied to use for the fabrication of surface-enhanced Raman scattering (SERS) active substrates for practical application in sensors. SERS is a well-known effect, initially discovered in 1970s and since then is under great debate in direction of understanding to develop a variety of SERS active substrates. The development of SERS substrates was further accelerated by the convergence of technologies (photonics and nanoscience) and now detection of a wide range of chemical and biological analytes is possible with detection sensitivity down to a single molecule [1]. At present, SERS is considered as one of the fast detection methods for applications in food, medicines and chemical analysis [2]. The SERS substrates are now routinely uses for both chemical and biological detection. SERS is based on an effect, promoted with the aid of surface plasmon resonance (SPR), a collective oscillation of the surface conduction electrons of a plasmonic metal NP such as silver (Ag) and gold ( $\mathrm{Au})$. Functional molecules such as graphene oxide have also been used as a scaffold to encapsulate the targets to promote SERS performance of analytes without strong substrate interactions [3].

Besides the enhanced ability of the substrate, interaction between the analyte and the substrate used is significantly important since only the molecules in close proximity of the hot-sites of the surface could give enhanced SERS signals. Due to biocompatibility and large scattering cross section, Ag nanoparticles (NPs) based SERS substrates are extensively used. The SERS performance highly depends on shape, size and distribution of the NPs on the solid surface [4]. The size of metal NPs further influence the local electromagnetic mechanism (EM) enhancement, and hence the SERS effect. In a suitable range, the local EM enhancement 
increases through inelastic scattering with increasing particle size [5]. Various types of SERS substrates have been tested including metal colloids, nanospheres, nanorods and nanowires for different detection purposes [6,7]. To ensure a homogeneous SERS response, uniformly-distributed plasmonic metal NPs are strongly proposed. Recently, particulate Ag solid films were proposed and used for SERS activity [8]. So, to produce SERS active substrates by a fast and feasible method with openness of flexibility of the substrates, is now in great demand for industrial scale application. Here, we propose a novel approach for the fabrication of SERS substrates, based on atmospheric dielectric barrier discharge (DBD) plasma. The method was recently tested to make plasmon active surface and worked very well [9].

In this method, Ag or Au plasmonic NP aerosols are produced by ablation process at atmospheric gas pressure and a flowing plasma jet from a DBD plasma source is combined with ablation to drive the aerosols towards the positioned substrate. The landed NP aerosols carried by the plasma jet, makes a SERS active surface and could be directly used for detection purposes. For the proposed method, we have noticed that for the same inter-electrode distance, driven AC voltage and different gas flow rates, the length of the ejaculated plasma stream is different and leads to different interactions with the substrate. For these interactions, the landed material forms different morphologies and features. Cluster and agglomerates formation was observed with the jet ignited at different gas flow rates and the substrate position [9]. Recently, Khan et.al applied a similar approach but used a gas jet at 4-10 litres per min to transport the NP aerosol of Ag target. The NP film-based SERS substrate prepared by atmospheric pulsed laser deposition (APLD) method were used for chemical detection of organic dye aqueous solution and detection sensitivity of the film was enhanced by annealing in air [10]. In the DBD plasma method, the formation of NP film was different when operated in flowing argon and helium. With argon plasma, the NPs were aggregated in clusters of mean size of $90 \mathrm{~nm}$ size while with helium plasma, two populations of NPs were observed: one of rounded particles with a mean size of $26 \mathrm{~nm}$ and the other of faceted particles with a mean size $160 \mathrm{~nm}$.

In another arrangement of the plasma method, the NP aerosols were flowed through the active region of the plasma column between the electrodes and subsequently entrained in the plasma jet for deposition to a remote substrate to make SERS active surface. In this case the deposited material was composed of bigger particulate structure, different from the approach where the NP aerosol was directly entrained in the plasma stream. One thing was quite obvious that with the DBD plasma, the deposition rate was significantly higher compared to a gas flow as reported in ref. 10. We expect that increase in the deposition rate by turning on the DBD plasma is due to the well-known phenomenon called electro-hydrodynamic gas pumping or the electric wind where ions in a weekly ionized plasma impart a bulk momentum to the gas and gas flow is accelerated. Based on the experimental parameters, agglomerated or sparsely distributed NPs could be obtained. This could give different SERS enhancement and detection sensitivity and would lead to the fabrication of different SERS substrates. In the DBD plasma assist APLD method, the formation of NP film strongly to depend on length of the plasma jet and is different for the same flow rate of Ar and He. Hence, the formation mechanism of the plasma jet is different for both $\mathrm{Ar}$ and $\mathrm{He}$ and would result in different morphologies and particulate structures that could lead to a different SERS enhancement and detection sensitivity. Several rooms are still open to further careful study in this direction to devise effective experimental approaches to further improve and develop this method.

\section{Conclusion}

A new and versatile DBD plasma-based method was introduced for fabrication of SERS substrates with different detection sensitivity for practical application. The DBD plasma increases the deposition rate and lead to the formation of different morphological features and hence SERS active surfaces with different detection sensitivity. The method is flexible to make SERS substrates with an open choice of selection the substrates such as glass, silicon, and filter paper. The approach will bring the fabrication method of SERS substrate one step closer for practical application. The uptake of the newly introduced DBD plasma assisted APLD method is quite fast and seems that in near future the method will prevail to combat the coexisting chemical and physical methods in several aspects. Further investigation is required to discern the physics in play.

\section{Acknowledgement}

This work was done in school of physics, Trinity College Dublin (TCD), the University of Dublin, Ireland, and supported by Science Foundation Ireland (SFI) under Investigator Project 12/IP/1662.

\section{References}

1. EC Le Ru, PG Etchegoin (2012) Single-Molecule Surface-Enhanced Raman Spectroscopy. Annual Review of Physical Chemistry 63: 65-87.

2. YS Li, JS Church (2014) Raman spectroscopy in the analysis of food and pharmaceutical nanomaterials. Journal Food and Drug Analysis 22(1): $29-48$.

3. H Zheng, D Ni, Z Yu, P Liang (2017) Preparation of SERS-active substrates based on graphene oxide/silver nanocomposites for rapid zdetection of l-Theanine. Food Chemistry 15: 511-516.

4. KL Kelly, E Coronado, LL Zhao, GC Schatz (2003) The optical properties of metal nanoparticles: The influence of size shape and dielectric environment. Journal of Physical Chemistry B 107(3): 668-677.

5. KG Stamplecoskie, JC Scaiano, VS Tiwari, H Anis (2011) Optimal size of silver nanoparticles for surface-enhanced Raman spectroscopy. Journal of Physical of Chemistry C 115(5): 1403-1409.

6. ND Israelsen, C Hanson, E Vargis (2015) Nanoparticle Properties and Synthesis Effects on Surface-Enhanced Raman Scattering Enhancement Factor: An Introduction. Scientific world journal 2015: 124582.

7. R Stiufiuc, C Iacovita, CM Lucaciu, G Stiufiuc, AG Dutu, et al. (2013) SERSActive Silver Colloids Prepared by Reduction of Silver Nitrate with ShortChain Polyethylene Glycol. Nanoscale Research Letters 8(1): 47.

8. CA Smyth, I Mirza, J G Lunney, E M McCabe (2013) Surface-enhanced 
Raman spectroscopy (SERS) using Ag nanoparticle films produced by pulsed laser deposition. Applied Surface Science 264(1): 31-35.

9. TM Khan, A Pokle, JG Lunney (2018) Atmospheric pulsed laser deposition of plasmonic nanoparticle films of silver with flowing gas and flowing atmospheric plasma. Applied Physics A 124: 265301.

\section{ISSN: 2574-1241}

DOI: 10.26717.BJSTR.2019.14.002544

Taj Muhammad Khan. Biomed J Sci \& Tech Res

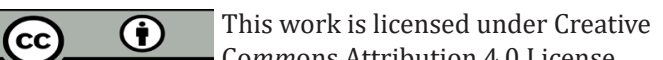

Submission Link: https://biomedres.us/submit-manuscript.php
10. TM Khan, MA Mujawar, KE Siewerska, A Pokle, T Donnelly, et al. (2017) Atmospheric pulsed laser deposition and thermal annealing of plasmonic silver nanoparticle films Nanotechnology 28: 445601.

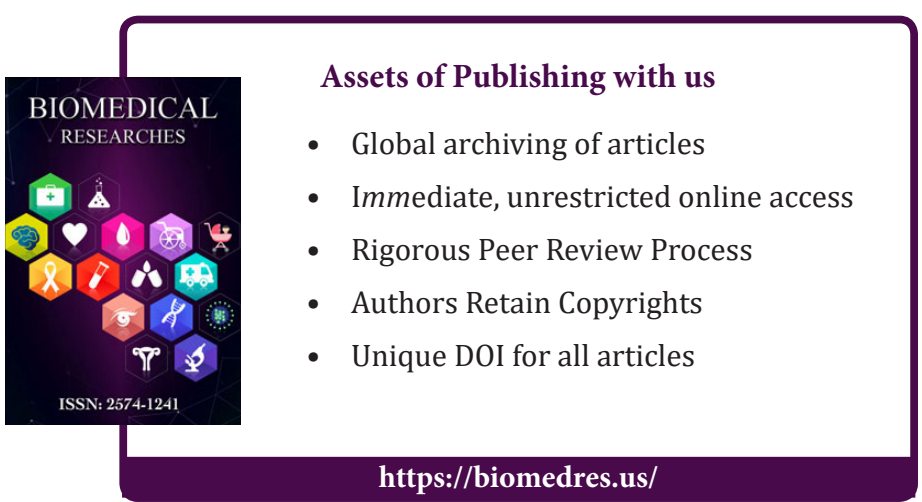

